\title{
A VECTORIAL SLEPIAN TYPE INEQUALITY. APPLICATIONS
}

\section{B. KHAOULANI}

(Communicated by William J. Davis)

\begin{abstract}
We prove a new inequality for Gaussian processes; this inequality implies the Chevet's inequality and Gordon's inequalities. Some remarks on Gaussian proofs of Dvoretzky's theorem are given.
\end{abstract}

\section{INTRODUCTION}

Let $\left\{g_{i, k}\right\} \quad(1 \leq i \leq n, 1 \leq k \leq d),\left\{h_{k}\right\}_{1}^{d}$, and $\left\{g_{i}\right\}_{1}^{n}$ denote independent sets of orthonormal Gaussian random variables. Let $E$ and $F$ be Banach spaces, $\left\{f_{k}\right\}_{k=1}^{d} \subset F$ and $\left\{x_{i}^{*}\right\}_{i=1}^{n} \subset E^{*}$. Let $T(\omega)=\sum_{i=1}^{n} \sum_{k=1}^{d} g_{i, k}(\omega) x_{i}^{*} \otimes f_{k}$ be a random operator from $E$ to $F$. The Chevet inequality says [Cv]

$$
\begin{aligned}
\mathbb{E}\left(\max _{\|x\|_{E}=1}\left\|T_{\omega} x\right\|\right) \leq \sqrt{2}( & \varepsilon_{2}\left(x_{1}^{*}, \ldots, x_{n}^{*}\right) \mathbb{E}\left(\left\|\sum_{k=1}^{d} h_{k} f_{k}\right\|\right) \\
& \left.+\varepsilon_{2}\left(f_{1}, \ldots, f_{d}\right) \mathbb{E}\left(\left\|\sum_{i=1}^{n} g_{i} x_{i}^{*}\right\|_{E^{*}}\right)\right),
\end{aligned}
$$

where

$$
\varepsilon_{2}\left(x_{1}^{*}, \ldots, x_{n}^{*}\right)=\sup \left\{\left(\sum_{1 \leq i \leq n} x_{i}^{*}(x)^{2}\right)^{1 / 2} ;\|x\|_{E} \leq 1\right\}
$$

and

$$
\varepsilon_{2}\left(f_{1}, \ldots, f_{d}\right)=\sup \left\{\left(\sum_{1 \leq k \leq d} y^{*}\left(f_{k}\right)^{2}\right)^{1 / 2} ;\left\|y^{*}\right\|_{F} \leq 1\right\} .
$$

Later, Gordon proved an inequality in the opposite direction:

$$
\begin{aligned}
& \inf _{\|x\|_{E}=1}\left\{\left(\sum_{i=1}^{n} x_{i}^{*}(x)^{2}\right)^{1 / 2}\right\} \mathbb{E}\left(\left\|\sum_{k=1}^{d} h_{k} f_{k}\right\|\right)-\varepsilon_{2}\left(f_{1}, \ldots, f_{d}\right) \mathbb{E}\left(\left\|\sum_{i=1}^{n} g_{i} x_{i}^{*}\right\|_{E^{*}}\right) \\
& \leq \mathbb{E}\left(\min _{\|x\|_{E}=1}\left\|T_{\omega} x\right\|\right) .
\end{aligned}
$$

Received by the editors July $25,1991$.

1991 Mathematics Subject Classification. Primary 46B09.

This work was supported by University of Paris VII, URA 1321, and E.N.I.M. Rabat. 
He also showed that the constant $\sqrt{2}$ in $(1.1)$ can be replaced by 1 (see [G1]). Our aim is to deduce these inequalities from a general Gaussian inequality for Gaussian processes.

\section{BASIC INEQUALITIES}

Let $(\Omega, \mathscr{A}, \mathbb{P})$ be a probability space and $X$ a canonical $\mathbb{R}^{d}$-valued Gaussian random vector (i.e., with covariance matrix equal to $\mathrm{Id}_{d}$ ). We define two Gaussian processes as follows. For $n \geq 1$, let $B_{2}^{n}$ be the closed unit ball of $l_{2}^{n}$ and $S^{n-1}$ its unit sphere. For $x=\left(x^{1}, \ldots, x^{n}\right) \in \mathbb{R}^{n}$, let $\|x\|_{2}=$ $\left(\sum_{i=1}^{n}\left(x^{i}\right)^{2}\right)^{1 / 2}$ and let $X_{1}, \ldots, X_{n}$ be $n$ independent copies of $X$, independent of $X$. Let $\left\{g_{1}, \ldots, g_{n}\right\}$ be a set of orthonormal Gaussian random variables independent of $\left\{X, X_{1}, \ldots, X_{n}\right\}$. Let

$$
X_{x}=\sum_{i=1}^{n} x^{i} X_{i} \text { and } g_{x}=\sum_{i=1}^{n} x^{i} g_{i}
$$

We shall prove the following inequality.

Theorem 2.1. Let $A \subset B_{2}^{n}$. Let $F_{x}: \mathbb{R}^{d} \rightarrow \mathbb{R}$ be a family of 1-Lipschitz functions indexed by $x \in A$. Then the Gaussian processes $\left\{X_{x}\right\}_{x \in A}$ and $\left\{g_{x}\right\}_{x \in A}$ satisfy

$$
\mathbb{E} \max _{x \in A} F_{x}\left(X_{x}\right) \leq \mathbb{E} \max _{x \in A}\left\{F_{x}\left(\|x\|_{2} X\right)+g_{x}\right\} .
$$

Corollary 2.1. Let $A \subset B_{2}^{n}$, and let $\|\cdot\| \|$ be a norm on $\mathbb{R}^{d}$ such that $\forall x \in \mathbb{R}^{d}$, \|\|$x\|\leq\| x \|_{2}$. Then the processes $\left\{X_{x}\right\}_{x \in A}$ and $\left\{g_{x}\right\}_{x \in A}$ verify

$$
\begin{aligned}
\min _{x \in A}\|x\|_{2}\left|\left\|X|\||-\mathbb{E} \max _{x \in A} g_{x}\right.\right. & \leq \mathbb{E} \min _{x \in A}\left|\left\|X_{x}\right\|\right| \leq \mathbb{E} \max _{x \in A}\left\|\left|X_{x} \|\right|\right. \\
& \leq \mathbb{E}|\|X\||+\mathbb{E} \max _{x \in A} g_{x} .
\end{aligned}
$$

Proof. For the right-hand side inequality put $F_{y}(x)=\||x|\|$, and for the lefthand side inequality put $F_{y}(x)=-|\|x|\||$.

Corollary 2.2. Let $X$ be a canonical $\mathbb{R}^{d}$-valued Gaussian random vector, with $X_{x}$ and $g_{x}$ as defined in (2.1). Let $A \subset S^{n-1}, F$ a 1-Lipschitz function on $\mathbb{R}^{d}$, and $\mu=\mathbb{E} F(X)$. Then the processes $\left\{X_{x}\right\}_{x \in A}$ and $\left\{g_{x}\right\}_{x \in A}$ verify

$$
\mathbb{E} \max _{x \in A}\left|F\left(X_{x}\right)-\mu\right| \leq \mathbb{E}|F(X)-\mu|+\mathbb{E} \max _{x \in A} g_{x} \leq 1+\mathbb{E} \max _{x \in A} g_{x}
$$

Proof. For the first inequality, take $G(\cdot)=|F(\cdot)-\mu|$, which is a 1-Lipschitz function; for the second, we use a well-known Poincaré-type inequality, that is,

$$
\mathbb{E}|f(X)-\mathbb{E}(f(X))|^{2} \leq \mathbb{E}\|\nabla f(X)\|_{2}^{2}
$$

for $X$ as above and all 1-Lipschitz functions $f$ on $\mathbb{R}^{d}[\mathrm{P}, \mathrm{C}]$.

Next we show how the Gordon inequalities follow from inequality (2.3). Indeed, let $u: \mathbb{R}^{d} \rightarrow F, u\left(\sum_{k=1}^{d} \alpha^{k} e_{k}\right)=\sum_{k=1}^{d} \alpha^{k} f_{k}$, and $v: E \rightarrow l_{n}^{2}, v(x)=$ $\left(x_{1}^{*}(x), \ldots, x_{n}^{*}(x)\right)$. We have $\|u\|=\varepsilon_{2}\left(f_{1}, \ldots, f_{d}\right)$ and $\|v\|=\varepsilon_{2}\left(x_{1}^{*}, \ldots, x_{n}^{*}\right)$. Let $X=\sum_{k=1}^{d} h_{k} e_{k}$, and for $1 \leq i \leq n$ let $X_{i}=\sum_{k=1}^{d} g_{i k} e_{k}$. Then $X$ is an $\mathbb{R}^{d}$ valued canonical Gaussian vector and $X_{1}, \ldots, X_{n}$ are $n$ independent copies of $X$, independent of $X$. Then $u\left(X_{v(x)}(\omega)\right)=T_{\omega}(x)$, so the rest of the proof is as in Corollary 2.1 with $A=v\left(S_{E}\right)$, where $S_{E}$ is the unit sphere of $E$ and $\||\alpha|\|=\|u(\alpha)\|$. 
Before proving Theorem 2.1, we get a vectorial Slepian type inequality, from which we deduce Theorem 2.1 (see Theorem 2.2).

We define some notation. For $x=\left(x_{i}\right), y=\left(y_{i}\right)$ in $\mathbb{R}^{d}, x \otimes y$ denotes the matrix $\left(x_{i} y_{i}\right)_{1 \leq i, j \leq d}$, and for $u, v \in \mathbb{R}^{d}$, define $x \otimes y[u, v]$ as $\langle u, x \otimes y(v)\rangle=$ $\langle x, u\rangle\langle y, v\rangle$ and $\|\cdot\|_{\mathscr{L}\left(R^{d}\right)}$ as the operator norm.

Theorem 2.2. Let $\left\{X_{t}\right\}$ and $\left\{Y_{t}\right\}, t \in T$, be two families of Gaussian vectors with values in $\mathbb{R}^{d}$, let $\left\{g_{t}\right\}$ be a family of Gaussian random variables independent of $\left\{X_{t}\right\}$ and $\left\{Y_{t}\right\}$, and suppose

(i) $\operatorname{dist}\left(X_{t}\right)=\operatorname{dist}\left(Y_{t}\right)$ for all $t \in T$,

(ii) $\left\|\mathbb{E}\left(X_{t} \otimes X_{s}-Y_{t} \otimes Y_{s}\right)\right\|_{\mathscr{L}\left(\mathbb{R}^{d}\right)} \leq \frac{1}{2} \mathbb{E}\left|g_{t}-g_{s}\right|^{2}$ for all $s, t$ in $T$.

Let $F_{t}, t \in T$, be a family of real 1-Lipschitz functions on $\mathbb{R}^{d}$. Then

$$
\mathbb{E} \sup _{t} F_{t}\left(X_{t}\right) \leq \mathbb{E} \sup _{t}\left\{F_{t}\left(Y_{t}\right)+g_{t}\right\}
$$

Proof. We may clearly assume without loss of generality that the two processes $\left\{X_{t}, t \in T\right\}$ and $\left\{Y_{t}, t \in T\right\}$ are independent and, also by a standard approximation argument, that the $F_{t}$ are 1-Lipschitz and twice differentiable.

It is clear that we just need to prove the inequality for finite sets $X_{1}, \ldots, X_{N}$, $Y_{1}, \ldots, Y_{N}(N \geq 1)$. Fix $X_{1}, \ldots, X_{N}$ and $Y_{1}, \ldots, Y_{N}$, and prove that

$$
\mathbb{E} \max _{1 \leq i \leq N}\left\{F_{i}\left(X_{i}\right)\right\} \leq \mathbb{E} \max _{1 \leq i \leq N}\left\{F_{i}\left(Y_{i}\right)+g_{i}\right\}
$$

For $\theta \in[0, \pi / 2]$ let

$$
Z(\theta)=\left(\cos (\theta) X_{1}+\sin (\theta) Y_{1}, \sin (\theta) g_{1} ; \ldots ; \cos (\theta) X_{N}+\sin (\theta) Y_{N}, \sin (\theta) g_{N}\right)
$$

where $Z(\theta)$ is an $\left(\mathbb{R}^{d+1}\right)^{N}$-valued Gaussian vector, with

$$
Z(0)=\left(X_{1}, 0 ; \ldots ; X_{N}, 0\right) \text { and } Z(\pi / 2)=\left(Y_{1}, g_{1} ; \ldots ; Y_{N}, g_{N}\right) ;
$$

a vector $(y, z)$ of $E=\left(\mathbb{R}^{d+1}\right)^{N}$ will be denoted by

$$
(y, z)=\left(\left(y_{i} ; z_{i}\right)\right)_{1 \leq i \leq N} \quad \text { where } y_{i} \in \mathbb{R}^{d} \text { and } z_{i} \in \mathbb{R} .
$$

We prove first the following lemma.

Lemma 2.1. Let $F: \mathbb{R}^{(d+1) N} \rightarrow \mathbb{R}^{N}, F(y, z)=\left(F_{1}\left(y_{1}\right)+z_{1}, \ldots, F_{N}\left(y_{N}\right)+z_{N}\right)$ where $F_{1}, \ldots, F_{N}$, are 1-Lipschitz twice differentiable on $\mathbb{R}^{d}$, and $G: \mathbb{R}^{N} \rightarrow$ $\mathbb{R}$ be a twice differentiable function such that $\exists k_{1}, k_{2}$, such that $|G(\cdot)| \leq$ $k_{1} e^{k_{2}\|\cdot\|_{2}},\left|\partial G(\cdot) / \partial \alpha_{i}\right| \leq k_{1} e^{k_{2}\|\cdot\|_{2}}$, and $\left|\partial^{2} G(\cdot) / \partial \alpha_{i} \partial \alpha_{j}\right| \leq k_{1} e^{k_{2}\|\cdot\|_{2}}$ for all $i, j=1, \ldots, N$. Put $\varphi=G \circ F$ and

$$
h(\theta)=\mathbb{E} \varphi(Z(\theta)) .
$$

Suppose

$$
\forall i, j, i \neq j, \quad \frac{\partial^{2} G}{\partial \alpha_{i} \partial \alpha_{j}} \leq 0
$$

and

$$
\forall j=1, \ldots, N \quad \sum_{i=1}^{N} \frac{\partial^{2} G}{\partial \alpha_{i} \partial \alpha_{j}}=0
$$


Then $h(\theta)$ is increasing, therefore,

$\mathbb{E} G\left(F_{1}\left(X_{1}\right), \ldots, F_{N}\left(X_{N}\right)\right)=h(0) \leq h(\pi / 2)=\mathbb{E} G\left(F_{1}\left(Y_{1}\right)+g_{1}, \ldots, F_{N}\left(Y_{N}\right)+g_{N}\right)$.

Proof of Lemma 2.1. Let $\varepsilon>0$, and let $\Lambda$ be an $\left(\mathbb{R}^{d+1}\right)^{N}$-valued canonical Gaussian vector independent of $\{Z(\theta) ; \theta \in] 0, \pi / 2[\}$. Let $Z_{\varepsilon}(\theta)=Z(\theta)+\varepsilon \Lambda$ so that $\Gamma_{\varepsilon}(\theta)=\Gamma(\theta)+\varepsilon^{2} I_{E}$, where $\Gamma(\theta)$ is the covariance matrix of $Z(\theta)$ and $\Gamma_{\varepsilon}(\theta)$ is the covariance matrix of $Z_{\varepsilon}(\theta)$. Thus

$$
\Gamma_{\varepsilon}(\theta) \rightarrow \Gamma(\theta) \text { as } \varepsilon \rightarrow 0 \text { so that } h_{\varepsilon}(\theta) \rightarrow h(\theta) \text { as } \varepsilon \rightarrow 0 .
$$

Remark that

$$
\forall(u, v) \in E \quad\left\langle(u, v), \Gamma_{\varepsilon}(\theta)(u, v)\right\rangle \geq \varepsilon^{2}\|(u, v)\|_{E}^{2} .
$$

Let $g_{\varepsilon}(y, z ; \theta)$ be the density function of $Z_{\varepsilon}(\theta)$. We will list the following well-known identities (see [G2, F, G1]):

$$
\begin{aligned}
g_{\varepsilon}(y, z ; \theta)=\frac{1}{(2 \pi)^{(d+1) N}} \int_{E} \exp \{ & i\langle(u, v) ;(y, z)\rangle \\
& \left.-\frac{1}{2}\left\langle(u, v), \Gamma_{\varepsilon}(\theta)(u, v)\right\rangle\right\} d u d v
\end{aligned}
$$

where $d u=d u_{1} \cdots d u_{N}, d u_{i}=d u_{i, 1} \cdots d u_{i, d}$, and $d v=d v_{1} \cdots d v_{N}$;

$$
\begin{gathered}
h_{\varepsilon}(\theta)=\int_{E} \varphi(y, z) g_{\varepsilon}(y, z, \theta) d y d z \quad\left[=\mathbb{E} \varphi\left(Z_{\varepsilon}(\theta)\right)\right] ; \\
h_{\varepsilon}^{\prime}(\theta)=\int_{E} \varphi(y, z) \frac{\partial}{\partial \theta} g_{\varepsilon}(y, z, \theta) d y d z ; \\
\frac{\partial}{\partial \theta} g_{\varepsilon}(x, \theta)=\frac{1}{2} \sum_{i, j=1}^{(d+1) N} \frac{d}{d \theta} \gamma_{i, j}^{\varepsilon}(\theta) \frac{\partial^{2}}{\partial x_{i} \partial x_{j}} g_{\varepsilon}(x, \theta)
\end{gathered}
$$

where $x=(y, z)$ and $\Gamma_{\varepsilon}(\theta)=\left(\gamma_{i, j}^{\varepsilon}(\theta)\right)_{1 \leq i, j \leq N(d+1)}$. We compute $\Gamma_{\varepsilon}(\theta)$. We can write $\Gamma_{\varepsilon}(\theta)$ as a block matrix: $\Gamma_{\varepsilon}(\theta)=\left(\Gamma_{i, j}^{\varepsilon}(\theta)\right)_{1 \leq i \leq N}, 1 \leq j \leq N$ where

$$
\Gamma_{i, j}^{\varepsilon}(\theta)=\mathbb{E}\left[Z_{i}^{\varepsilon}(\theta) \otimes Z_{j}^{\varepsilon}(\theta)\right]
$$

where

$$
Z_{i}^{\varepsilon}(\theta)=\left(X_{i}(\theta)+Y_{i}(\theta)+\varepsilon \Lambda_{i}, \quad g_{i}(\theta)+\varepsilon \Lambda_{i}^{\prime}\right)
$$

where

$$
\begin{aligned}
& \Lambda=\left(\Lambda_{i}, \Lambda_{i}^{\prime}\right)_{1 \leq i \leq N}, \quad \Lambda_{i}=\left(\Lambda_{i}^{1}, \ldots, \Lambda_{i}^{d}\right), \\
X_{i}(\theta)= & \cos (\theta) X_{i}, \quad Y_{i}(\theta)=\sin (\theta) Y_{i}, \quad g_{i}(\theta)=\sin (\theta) g_{i} .
\end{aligned}
$$

Using the fact that $\left\{X_{1}, \ldots, X_{N}\right\},\left\{Y_{1}, \ldots, Y_{N}\right\}$, and $\left\{g_{1}, \ldots, g_{N}\right\}$ are independent processes, we find that

$$
\Gamma_{i, j}^{\varepsilon}(\theta)=\left[\begin{array}{cc}
A_{i, j}(\theta)+\varepsilon^{2} \operatorname{Id}_{d} \delta i, j & 0 \\
0 & B_{i, j}^{\varepsilon}(\theta)
\end{array}\right]
$$

where $A_{i, j}(\theta)$ is a $d \times d$ matrix and $B_{i, j}^{\varepsilon}(\theta)$ is a scalar such that

$$
\begin{aligned}
& A_{i j}(\theta)=\cos ^{2}(\theta) \mathbb{E}\left(X_{i} \otimes X_{j}\right)+\sin ^{2}(\theta) \mathbb{E}\left(Y_{i} \otimes Y_{j}\right), \\
& B_{i j}^{\varepsilon}(\theta)=\sin ^{2}(\theta) \mathbb{E} g_{i} g_{j}+\varepsilon^{2} \delta_{i, j}
\end{aligned}
$$


where $\delta_{i, j}=1$ if $i=j$, and 0 if $i \neq j$. A simple computation gives

$$
\begin{aligned}
& \left\langle(u, v) ; \Gamma_{\varepsilon}(\theta)(u, v)\right\rangle \\
& =\sum_{i=1}^{N} \sum_{j=1}^{N}\left[\left\langle u_{i}, A_{i, j}(\theta) u_{j}\right\rangle+\varepsilon^{2}\left\langle u_{i}, u_{j}\right\rangle\right]+\sum_{i=1}^{N} \sum_{j=1}^{N} B_{i, j}^{\varepsilon}(\theta) v_{i} \cdot v_{j} .
\end{aligned}
$$

Considering $\partial^{2} g_{\varepsilon}(y, z ; \theta) / \partial y_{i} \partial y_{j}$ as a $d \times d$ matrix for each $i, j$ gives

$$
\begin{aligned}
\frac{\partial}{\partial \theta} g_{\varepsilon}(y, z ; \theta)= & \frac{1}{2} \sum_{i, j=1}^{N} \operatorname{trace}\left(\frac{\partial^{2}}{\partial y_{i} \partial y_{j}} g_{\varepsilon}(y, z ; \theta) \frac{d}{d \theta} A_{i, j}^{\varepsilon}(\theta)\right) \\
& +\frac{1}{2} \sum_{i, j=1}^{N} \frac{d}{d \theta} B_{i, j}^{\varepsilon}(\theta) \frac{\partial^{2}}{\partial z_{i} \partial z_{j}} g_{\varepsilon}(y, z ; \theta) ;
\end{aligned}
$$

but

$$
h_{\varepsilon}^{\prime}(\theta)=\int \varphi(y, z) \frac{\partial}{\partial \theta} g_{\varepsilon}(y, z, \theta) d y d z .
$$

Let $M_{i, j}=\mathbb{E} Y_{i} \otimes Y_{j}-\mathbb{E} X_{i} \otimes X_{j}$. We get

$$
\begin{aligned}
h_{\varepsilon}^{\prime}(\theta)=\frac{\sin 2 \theta}{2} \int_{E}\left\{\sum_{i, j=1}^{N} \operatorname{trace}\right. & \left(\frac{\partial^{2} \varphi(y, z)}{\partial y_{i} \partial y_{j}} \cdot M_{i, j}\right) \\
& \left.+\sum_{i, j=1}^{N} \frac{\partial^{2} \varphi(y, z)}{\partial z_{i} \partial z_{j}} \mathbb{E} g_{i} g_{j}\right\} g_{\varepsilon}(y, z, \theta) d y d z .
\end{aligned}
$$

Since $\operatorname{dist}\left(X_{i}\right)=\operatorname{dist}\left(Y_{i}\right)$ for all $i$, we get $M_{i, i}=0$; hence, we have, for $\varphi=G \circ F$,

$$
\begin{aligned}
h_{\varepsilon}^{\prime}(\theta)=\frac{\sin 2 \theta}{2} \int\left\{\sum_{i \neq j}^{N}\right. & \operatorname{tr}\left(\frac{\partial^{2} G \circ F}{\partial y_{i} \partial y_{j}} \cdot M_{i, j}\right) \\
& \left.+\sum_{i, j=1}^{N}\left(\frac{\partial^{2} G \circ F}{\partial z_{i} \partial z_{j}}\right) \mathbb{E} g_{i} g_{j}\right\} g_{\varepsilon}(y, z ; \theta) d y d z .
\end{aligned}
$$

A simple computation gives, for all $i \neq j$,

$$
\frac{\partial^{2} G \circ F}{\partial y_{i} \partial y_{j}}=\frac{\partial^{2} G}{\partial \alpha_{i} \partial \alpha_{j}} \circ F \cdot \nabla F_{i}\left(y_{i}\right) \otimes \nabla F_{j}\left(y_{j}\right)
$$

and

$$
\frac{\partial^{2} G \circ F}{\partial z_{i} \partial z_{j}}=\frac{\partial^{2} G}{\partial \alpha_{i} \partial \alpha_{j}} \circ F \text { for all } i, j .
$$

Condition (2.7) gives

$$
\frac{\partial^{2} G}{\partial \alpha_{i}^{2}}=-\sum_{j=1, j \neq i}^{N} \frac{\partial^{2} G}{\partial \alpha_{i} \partial \alpha_{j}} \text { for all } i, j,
$$


so

$$
\begin{aligned}
& h_{\varepsilon}^{\prime}(\theta)=\frac{\sin 2 \theta}{2} \int\left\{\sum_{i \neq j}^{N} \operatorname{tr}\left(\frac{\partial^{2} G(F(y, z))}{\partial y_{i} \partial y_{j}} \cdot M_{i, j}\right)+\sum_{i \neq j}^{N} \frac{\partial^{2} G(F(y, z))}{\partial z_{i} \partial z_{j}} \mathbb{E} g_{i} g_{j}\right. \\
& \left.+\sum_{i=1}^{N} \frac{\partial^{2} G(F(y, z))}{\partial z_{i}^{2}} \mathbb{E} g_{i}^{2}\right\} g_{\varepsilon}(y, z ; \theta) d y d z \\
& =\frac{\sin 2 \theta}{2} \int\left\{\sum _ { i \neq j } ^ { N } \left(\operatorname{tr}\left(\frac{\partial^{2} G(F(y, z))}{\partial y_{i} \partial y_{j}} \cdot M_{i, j}\right)\right.\right. \\
& +\left(\mathbb{E} g_{i} g_{j}-\frac{1}{2}\left[\mathbb{E} g_{i}^{2}+\mathbb{E} g_{j}^{2}\right]\right) \\
& \left.\left.\times \frac{\partial^{2} G(F(y, z))}{\partial \alpha_{i} \partial \alpha_{j}}\right)\right\} g_{\varepsilon}(y, z ; \theta) d y d z \\
& =\frac{\sin 2 \theta}{2} \int\left\{\sum _ { i \neq j } ^ { N } \left(\frac { \partial ^ { 2 } G ( F ( y , z ) ) } { \partial \alpha _ { i } \partial \alpha _ { j } } \left(\left\langle M_{i, j} \cdot \nabla F_{i}\left(y_{i}\right), \nabla F_{j}\left(y_{j}\right)\right\rangle\right.\right.\right. \\
& \left.\left.\left.-\frac{1}{2} \mathbb{E}\left|g_{i}-g_{j}\right|^{2}\right)\right)\right\} g_{\varepsilon}(y, z ; \theta) d y d z .
\end{aligned}
$$

Since $\left\|\vec{\nabla} F_{i}\left(y_{i}\right)\right\| \leq 1$,

$$
\left\langle M_{i, j}\left(\nabla F_{i}\left(y_{i}\right)\right), \nabla F_{j}\left(y_{j}\right)\right\rangle-\frac{1}{2} \mathbb{E}\left|g_{i}-g_{j}\right|^{2} \leq\left\|\left(M_{i, j}\right)\right\|_{\mathscr{L}\left(\mathbb{R}^{d}\right)}-\frac{1}{2} \mathbb{E}\left|g_{i}-g_{j}\right|^{2} \leq 0,
$$

so $h_{\varepsilon}^{\prime}(\theta) \geq 0$ and $\mathbb{E} G\left(F\left(Z_{\varepsilon}(0)\right)\right) \leq \mathbb{E} G\left(F\left(Z_{\varepsilon}(\pi / 2)\right)\right)$. Finally, letting $\varepsilon \rightarrow 0$, we get the result of Lemma 2.1 .

We now finish the proof of Theorem 2.2. The map max which assigns to each $\left(\alpha_{1}, \ldots, \alpha_{N}\right) \in \mathbb{R}^{N}$ the value $\max \left(\alpha_{1}, \ldots, \alpha_{N}\right)$ is slowly increasing and verifies (2.6) and (2.7) in distribution sense [G2]. So if we regularise max by convolution with a twice differentiable function $\psi_{k}$, which is supported by a ball of radius $1 / k$, we obtain a function $m_{k}$, which is 1 -Lipschitz and satisfies the above three conditions. By considering the functions $h_{k}(\theta)=\mathbb{E} m_{k} \circ F(Z(\theta))$, and by letting $k$ go to infinity, we find by Lebesgue's theorem that the function $\mathbb{E} \max \circ F(Z(\cdot))$ is increasing in $[0 ; \pi / 2]$. This completes the proof of Theorem 2.2 .

Proof of Theorem 2.1. We have $X_{x}=\sum_{i=1}^{n} x^{i} X_{i}$. Let $Y_{x}=\|x\|_{2} X$, where $x$ runs over a set $A \subset B_{n}^{2}$. Then $\operatorname{dist}\left(X_{x}\right)=\operatorname{dist}\left(Y_{x}\right)$. Take a finite set $\left\{a_{1}, \ldots, a_{N}\right\}$ in $A$; a simple computation gives

$$
M_{i, j}=\mathbb{E}\left(Y_{a_{i}} \otimes Y_{a_{j}}-X_{a_{i}} \otimes X_{a_{j}}\right)=\left(\left\|a_{i}\right\|_{2}\left\|a_{j}\right\|_{2}-a_{i} \cdot a_{j}\right) \operatorname{Id}_{d}
$$

where $a_{i} \cdot a_{j}$ is the scalar product. Moreover, $\mathbb{E}\left|g_{a_{i}}-g_{a_{j}}\right|^{2}=\left\|a_{i}-a_{j}\right\|_{2}^{2}$, the $F_{i}$ are 1-Lipschitz functions, so

$$
\begin{aligned}
\left\|M_{i, j}\right\|_{\mathscr{L}\left(\mathbf{R}^{d}\right)}-\frac{1}{2} \mathbb{E}\left|g_{a_{i}}-g_{a_{j}}\right|^{2} & =\left(\left\|a_{i}\right\|_{2}\left\|a_{j}\right\|_{2}-a_{i} \cdot a_{j}\right)-\frac{1}{2}\left\|a_{i}-a_{j}\right\|_{2}^{2} \\
& =-\frac{1}{2}\left(\left\|a_{i}\right\|_{2}-\left\|a_{j}\right\|_{2}\right)^{2} \leq 0 .
\end{aligned}
$$


Hence conditions (i) and (ii) of Theorem 2.2 are satisfied, and Theorem 2.1 is proved.

\section{FINAL REMARKS}

We give now a short proof of a result due to Milman.

Theorem 3.1 [M, Sc]. Let $\varepsilon>0, f: \mathbb{R}^{N} \rightarrow \mathbb{R}$ be a Lipschitz function with constant $L, X=\sum_{i=1}^{N} g_{i} e_{i}$ where $\left\{g_{i}\right\}_{1 \leq i \leq N}$ is a set of orthonormal Gaussian random variables and $\left\{e_{i}\right\}_{1 \leq i \leq N}$ is the canonical basis of $l_{2}^{N}$, and $\mu=\mathbb{E} f(X)$. Then there exists an operator $T: l_{2}^{n} \rightarrow \mathbb{R}^{N}$ with $n=[(\varepsilon \mu / L)(\varepsilon \mu / L-2)]$, such that

$$
|f(T x)-\mu| \leq \varepsilon \mu \text { for all } x \in S^{n-1} .
$$

Proof. Consider, as above, real-valued Gaussian operator $T_{\omega}=\sum_{i=1}^{n} \sum_{j=1}^{N} g_{i j} e_{i}^{*}$ $\otimes e_{j}$ from $l_{n}^{2}$ to $\mathbb{R}^{N}$,

$$
X_{i}=\sum_{j=1}^{N} g_{i, j} e_{j} \quad \text { and } \quad X_{x}=\sum_{i=1}^{n} x^{i} X_{i}
$$

where $x=\left(x^{i}, \ldots, x^{n}\right)$. Then $X_{x}(\omega)=T_{\omega} x$, and we have

$$
\begin{aligned}
\mathbb{P}\left(\left\{\omega / \exists x \in S^{n-1} ;\left|f\left(X_{x}\right)-\mu\right|>\varepsilon \mu\right\}\right) & =\mathbb{P}\left(\left\{\omega ; \sup _{x \in S^{n-1}}\left|f\left(X_{x}\right)-\mu\right|>\varepsilon \mu\right\}\right) \\
& \leq \frac{1}{\varepsilon \mu} \mathbb{E} \sup _{x \in S^{n-1}}\left|f\left(X_{x}\right)-\mu\right| .
\end{aligned}
$$

We apply Corollary 2 to get

$$
\begin{aligned}
& \mathbb{P}\left(\left\{\omega / \exists x \in S^{n-1} ;\left|f\left(X_{x}\right)-\mu\right|>\varepsilon \mu\right\}\right) \\
& \quad \leq \frac{1}{\varepsilon \mu}\left\{\mathbb{E}|f(X)-\mu|+L \mathbb{E} \sup _{x \in S^{n-1}} \sum_{j=1}^{n} x^{j} g_{j}\right\},
\end{aligned}
$$

and using the Poincaré-type inequality as in Corollary 2, we find that

$$
\begin{aligned}
\mathbb{P}\left(\left\{\omega / \exists x \in S^{n-1} ;\left|f\left(X_{x}\right)-\mu\right|>\varepsilon \mu\right\}\right) & \leq \frac{L}{\varepsilon \mu}\left[1+\mathbb{E} \sup _{x \in S^{n-1}} \sum_{j=1}^{n} x^{j} g_{j}\right] \\
& \leq \frac{L}{\varepsilon \mu}(1+\sqrt{n}) .
\end{aligned}
$$

We only need to choose $n$ such that this last expression is $<1$.

\section{ACKNOWLEDGMENT}

I express my warmest thanks to Professor B. Maurey for suggesting to me this question and for many stimulating and fruitful conversations.

\section{REFERENCES}

[C] L. Chen, An inequality for the multivariate normal distributions, J. Multivariate Anal. 12 (1982), 306-315.

[Cv] S. Chevet, Séries de variables aléatoires Gaussiennes à valeurs dans E $\widehat{\otimes}_{\varepsilon} F$. Application aux produits d'espaces de Wiener, Séminaire Maurey-Schwartz, exposé XiX 77/78. 
[F] X. M. Fernique, Régularité des trajectoires des fonctions aléatoires Gaussiennes, Lecture Notes in Math., vol. 480, Springer-Verlag, New York, 1974.

[G1] Y. Gordon, Some inequalities for Gaussian processes and applications, Israel J. Math. 50 (1985), 265-289.

[G2] _ Elliptically contourned distributions, Probab. Theory Related Fields 76 (1987), 429438.

[K] J. P. Kahane, Une inégalité de type Slepian et Gordon sur les processus Gaussiens, Israel J. Math. 55 (1986), 109-110.

[M] V. D. Milman, New proof of the theorem of Dvoretzky on sections of convex bodies, Funktsional. Anal. i Prilozhen. 5 (1971).

[P] G. Pisier, Probabilistic methods in the geometry of Banach spaces, Lecture Notes in Math, vol. 1206, Springer, New York, pp. 1-154.

[Sc] G. Schechtman, A remark concerning the dependence on $\varepsilon$ in Dvoretzksy's theorem, Lecture Notes in Math., vol. 1376, Springer, New York, pp. 274-277.

Equipe d’Analyse, Universite of Paris VII, 4 Place, Jussieu, 75230 Paris Cedex 05, FRANCE

Current address: E.N.I.M., Departement d'Informatique, B. P. 753, Rabat-Agdal, Morocco 